\title{
INGREDIENTES ALTERNATIVOS EN LA ALIMENTACIÓN DE CODORNICES
}

\author{
ALTERNATIVE INGREDIENTS IN FEED FOR QUAIL
}

Ferney Domingo Agudelo

Egresado de Medicina Veterinaria Zootecnia de la Universidad de los Llanos

Ferney.agudelo@unillanos.edu.co

Víctor Libardo Hurtado Nery

Ph.D. Ciencia Animal, Universidad de los Llanos

victor.hurtado@unillanos.edu.co

https://orcid.org/0000-0002-9890-5726

Diana Milena Torres Novoa

MSc. Ciencia Animal, Universidad Nacional Abierta y a Distancia (UNAD)

milena.torres@unad.edu.co

https://orcid.org/0000-0003-3394-791

\section{RESUMEN}

Contextualización: La incorporación de alimentos no convencionales en las dietas de codornices se realiza con propósito de reducir los costos de producción, mejorar la rentabilidad, sin afectar la calidad del huevo, entendida ésta como las características que influyen en la aceptabilidad por los consumidores.

Vacío de conocimiento: Potencial uso de alimentos alternativos y subproductos del procesamiento de arroz en los Llanos de Colombia como fuente energética para raciones de codornices, dado que la alimentación de las aves constituye una alta proporción de los costos totales de producción.

DOI: https://doi.org/10.22490/26653176.4303 
Propósito: Evaluar el desempeño productivo y algunas características de la calidad del huevo de codornices japonesas en fase inicial de postura utilizando ingredientes alternativos en sustitución parcial del maíz.

Metodología: Fueron utilizadas 250 codornices de 50 días de edad en un diseño experimental completamente al azar, con cinco tratamientos, cinco repeticiones y 10 aves por repetición, durante 16 semanas. Los tratamientos fueron dietas: convencional, con arroz partido, harina de arroz, afrecho de yuca y harina de plátano en sustitución del 50\% de maíz. El agua y raciones fueron suministradas a voluntad.

Resultados y conclusiones: El consumo diario de ración $(24,39 ; 24,11 ; 24,54 ; 24,01$ y $24,80 \mathrm{~g} /$ día $)$ y el peso del huevo $(10,2 ; 10,3 ; 10,2 ; 10,0$ y 10,2 gramos) no fueron influenciados $(p>0,05)$ por los ingredientes alternativos. Los alimentos alternativos influenciaron $(p<0,05)$ la producción de huevos $(78,63 ; 66,26 ; 60,75 ; 53,56$ y 58,87\%), los $\mathrm{kg}$ de ración por docena de huevos $(0,376 ; 0,440 ; 0,491 ; 0,545$ y $0,514 \mathrm{~kg})$ y por $\mathrm{kg}$ de huevo $(3,057 ; 3,561 ; 4,004 ; 4,529$ y $4,180 \mathrm{~kg})$.

Conclusiones: El arroz partido puede sustituir en 50\% al maíz, en la fabricación de raciones para codornices japonesas en fase inicial de postura sin afectar la producción de huevos, y el afrecho de yuca, las harinas de arroz y de plátano en sustitución al 50\% del maíz, proporcionan bajos índices de desempeño productivo.

Palabras-clave: alimento no convencional, Coturnix japónica, desempeño, producción de huevo (Fuentes: CAB)

\footnotetext{
ABSTRACT

Contextualization: The incorporation of unconventional foods in quail diets is carried out with the purpose of reducing production costs, improving profitability, without affecting the quality of the egg, understood as the characteristics that influence acceptability by consumers.

Knowledge gap: Potential use of alternative feeds and rice processing by-products in the Llanos de Colombia as an energy source for quail rations, given that poultry feeding constitutes a high proportion of total production costs.

Purpose: To evaluate the productive performance and some characteristics of the quality of the Japanese quail in the initial stage of laying, using alternative ingredients in partial substitution of corn.

Methodology: 250 50-day-old quails were used in a completely randomized experimental design, with five treatments, five repetitions and 10 birds per repetition, for 16 weeks. The treatments were diets: conventional, with broken rice, rice flour, cassava bran and banana flour replacing $50 \%$ corn. Water and rations were supplied ad libitum.
} 
Results and conclusions: Daily ration consumption (24.39, 24.11, 24.54, 24.01 and 24.80 $\mathrm{g} /$ day) and egg weight (10.2,10.3,10.2; 10.0 and 10.2 grams) were not influenced ( $p>$ $0.05)$ by the alternative ingredients. Alternative foods influenced $(p<0.05)$ the production of eggs $(78.63,66.26,60.75,53.56$ and $58.87 \%)$, the $\mathrm{kg}$ of ration per dozen eggs $(0.376$, $0.440 ; 0.491 ; 0.545$ and $0.514 \mathrm{~kg})$ and per $\mathrm{kg}$ of egg $(3.057 ; 3.561 ; 4.004 ; 4.529$ and 4.180 $\mathrm{kg})$.

Conclusions: Broken rice can substitute $50 \%$ for corn in the manufacture of rations for Japanese quail in the initial laying phase without affecting egg production, and cassava bran, rice and banana flours substitute $50 \%$. \% of corn, provide low indexes of productive performance.

Keywords: unconventional food; coturnix japonica; performance; egg production (Sources: CAB).

\section{RESUMEN GRÁFICO}

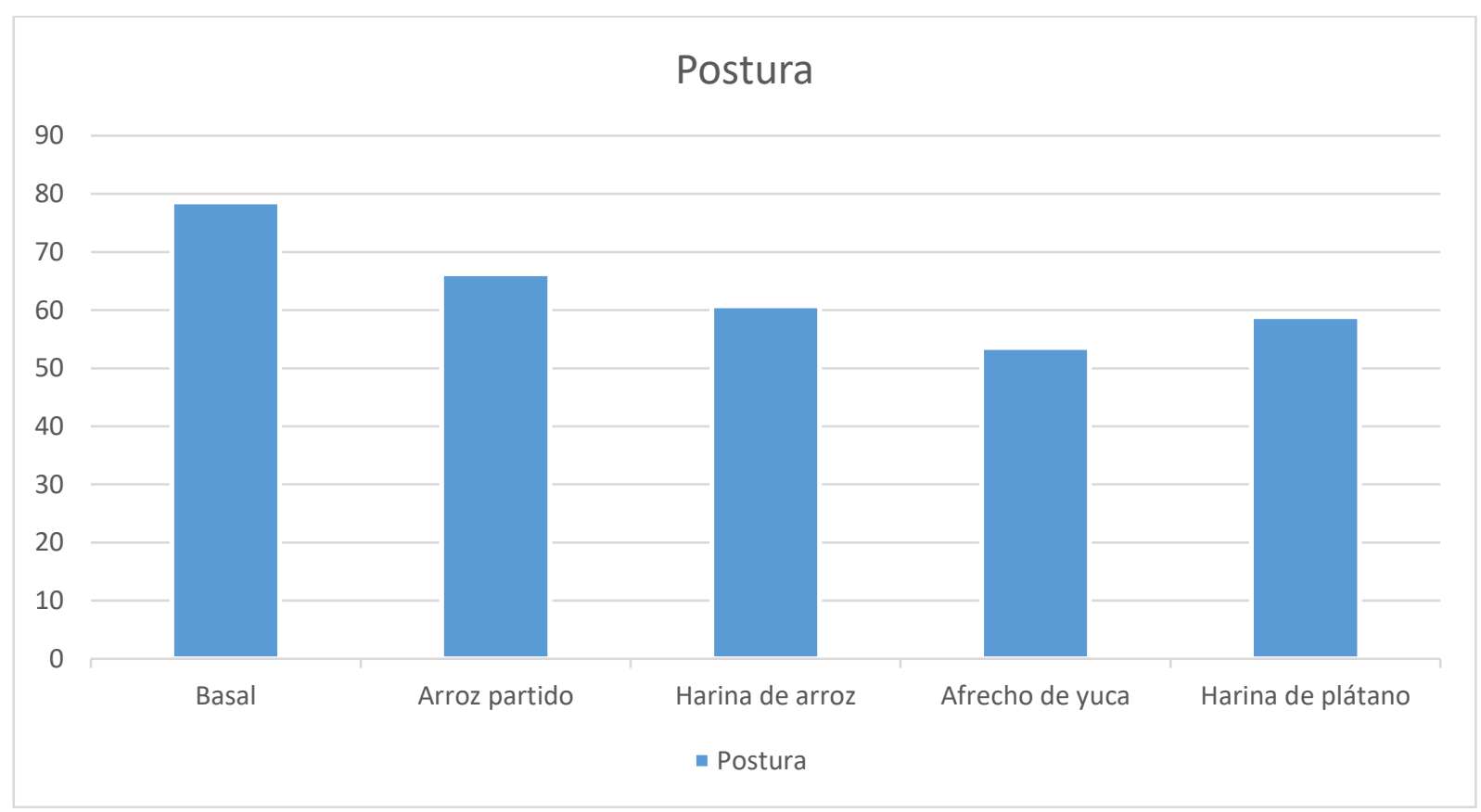

Fuente: Autores

\section{INTRODUCCIÓN}

La producción de codornices es una actividad que requiere poco espacio por ave para su alojamiento, en la alimentación, se puede incorporar alimentos alternativos atendiendo los requerimientos nutricionales de las aves, con propósito de reducir los costos de producción, 
mejorar la rentabilidad, sin afectar las características de calidad del huevo (Hrnčár et al., 2014). El valor de la alimentación en las aves compone en alta proporción los costos totales de producción, lo cual genera la necesidad de buscar alimentos alternativos

Los Llanos Orientales de Colombia son una despensa agrícola que permite obtener alimentos alternativos y subproductos del procesamiento de arroz, los cuales se pueden utilizar como fuente energética en raciones para codornices. Según Rostagno et al. (2017), el arroz partido y la harina de arroz contienen 3219 y $3152 \mathrm{kcal} / \mathrm{kg}$ de energía metabolizable respectivamente. El arroz partido constituye una alternativa para su utilización en la alimentación animal por el bajo costo (Gopinger et al., 2014; Rahman et al., 2019), permitiendo que estos coproductos del arroz puedan ser utilizados en la alimentación de aves en zonas cálidas (Farias et al., 2014).

El maíz es el cereal de mayor uso en la alimentación de aves por su alto contenido en almidón y grasa, como fuente de ácido linoleico ( $1,8 \%$ del total de extracto etéreo), ácido graso esencial en el metabolismo animal, especialmente en el aviar (Campos y Vega 2016) el maíz proporciona de 7 - 9\% de proteína, $3330 \mathrm{kcal} / \mathrm{kg}$ de energía metabolizable (Rostagno et al. 2017). Utilizando ingredientes alternativos en la alimentación de codornices, Degollado (2018) reportó $60,73 \%$ de postura con $5 \%$ de hoja de Moringa oleífera y $45,78 \%$ de proteína cruda

En consideración a lo expuesto anteriormente, este trabajo fue realizado con el objetivo de evaluar la producción de huevos, el consumo de ración y la conversión alimenticia de codornices japonesas en fase inicial de postura, utilizando arroz, harina de arroz, afrecho de yuca, harina de plátano en sustitución parcial al maíz de la dieta basal.

\section{MATERIALES Y MÉTODOS}

\section{Ubicación:}

El trabajo fue realizado en la Granja de la Universidad de los Llanos Km 12 Vía Apiay en el municipio de Villavicencio, Meta, Colombia, que se encuentra ubicado a 423 metros de altitud sobre el nivel del mar, con temperatura media de $27^{\circ} \mathrm{C}, 82 \%$ de humedad relativa, $3500 \mathrm{~mm}$ de precipitación anual (IGAC, 2021).

\section{Diseño experimental:}

El ensayo se realizó en un diseño experimental completamente al azar con cinco tratamientos, cinco repeticiones y diez aves por réplica para un total de 250 codornices de 50 días de edad. Las codornices fueron alojadas en jaulas de alambre galvanizado, con dimensiones de $1 \mathrm{~m}$ de largo $\times 0.65 \mathrm{~m}$ de ancho $\times 0.2 \mathrm{~m}$ de altura, que constan de cinco pisos con tres divisiones y capacidad de 10 aves por división, dotados de comederos lineales 
y bebederos automáticos con suministro de alimento y agua a voluntad. La composición de las raciones experimentales se presenta en la Tabla 1.

Tabla 1. Composición centesimal de las raciones experimentales.

\begin{tabular}{|c|c|c|c|c|c|}
\hline \multirow[b]{2}{*}{ Ingredientes } & \multicolumn{5}{|c|}{ Tratamientos } \\
\hline & $\begin{array}{l}\text { Dieta } \\
\text { basal }\end{array}$ & $\begin{array}{c}\text { Arroz } \\
\text { partido }\end{array}$ & $\begin{array}{c}\text { Harina de } \\
\text { arroz }\end{array}$ & $\begin{array}{l}\text { Afrecho } \\
\text { de Yuca }\end{array}$ & $\begin{array}{c}\text { Harina de } \\
\text { Plátano }\end{array}$ \\
\hline Maíz & 56,53 & 28,27 & 28,27 & 28,27 & 28,27 \\
\hline Torta de soya & 32.10 & 33,83 & 29,00 & 35,52 & 35,52 \\
\hline Arroz partido & 0,00 & 28,27 & 0,00 & 0,00 & 0,00 \\
\hline Harina de yuca & 0,00 & 0,00 & 0,00 & 28,27 & 0,00 \\
\hline Harina de arroz & 0,00 & 0,00 & 28,27 & 0,00 & 0,00 \\
\hline Harina de plátano & 0,00 & 0,00 & 0,00 & 0,00 & 28,27 \\
\hline Fosfato bicálcico & 1,35 & 1,35 & 1,00 & 1,20 & 1,20 \\
\hline Carbonato de calcio & 5,40 & 5,40 & 5,45 & 5,45 & 5,45 \\
\hline Aceite vegetal & 0.80 & 1,40 & 2,90 & 0,10 & 0,10 \\
\hline Premezcla de vitaminas & 0,50 & 0,50 & 0,50 & 0,50 & 0,50 \\
\hline Sal & 0,50 & 0,50 & 0,50 & 0,50 & 0,50 \\
\hline L-lisina & 0,05 & 0,03 & 0,04 & 0,02 & 0,02 \\
\hline DL Metionina & 0,18 & 0,16 & 0,17 & 0,17 & 0,17 \\
\hline Inerte & 2,54 & 2,58 & 3,90 & 0,00 & 0,00 \\
\hline Total & 100,00 & 100,00 & 100,00 & 100,00 & 100,00 \\
\hline \multicolumn{6}{|c|}{ Contenido nutricional de las raciones experimentales } \\
\hline Proteína bruta, \% & 19,20 & 19,20 & 19,20 & 18,430 & 18,43 \\
\hline Energía metabolizable, kcal/kg & 3000 & 3000 & 3000 & 3000 & 3000 \\
\hline Fósforo disponible, \% & 0,35 & 0,35 & 0,35 & 0.31 & 0.31 \\
\hline Calcio, \% & 2,50 & 2,50 & 2,50 & 2,50 & 2,50 \\
\hline Metionina, \% & 0,45 & 0,45 & 0,45 & 0,42 & 0,42 \\
\hline Lisina, \% & 1.00 & 1,00 & 1,00 & 1,00 & 1,00 \\
\hline
\end{tabular}

Fuente: Autores

La fase experimental tuvo una duración de 12 semanas, durante las cuales las aves recibían luz natural y artificial durante 14 horas diarias, las condiciones de temperatura y humedad relativa se monitorearon diariamente en horas de la mañana y en la tarde. Los parámetros evaluados fueron producción de huevos (\% ave/alojada/día), peso del huevo (g), consumo diario de ración ( $\mathrm{g}$ ) y conversión alimentar ( $\mathrm{kg}$ de ración/docenas de huevos y masa de huevo). Los ingredientes y las raciones experimentales fueron analizados en el Laboratorio de Nutrición Animal de la Universidad de los Llanos.

\section{Análisis Estadístico}

Para determinar la normalidad de los datos experimentales se aplicó la prueba de Shapiro Wilk. Los datos de desempeño fueron sometidos a análisis de varianza y comparación de medias por la prueba de Scheffe al $5 \%$ de probabilidad en el programa Statistix 8.0 (Analytical software, Tallahassee, 2015), según el siguiente modelo estadístico: 


\section{$Y \mathbf{i j}=\boldsymbol{\mu}+\mathbf{t i}+\varepsilon \mathbf{i j}$}

Yij = Respuesta de la variable (postura de huevos, consumo diario de ración, peso del huevo y conversión alimenticia bajo el tratamiento i.

$\boldsymbol{\mu}=$ Media general.

ti = Efecto del tratamiento i, siendo i Dieta basal, arroz partido, harina de arroz, afrecho de yuca, harina de plátano.

$\varepsilon \mathbf{i j}=$ Efecto residual.

\section{RESULTADOS Y DISCUSIÓN}

Los resultados de desempeño zootécnico de codornices alimentadas con ingredientes alternativos se presentan en la tabla 2. La sustitución parcial del maíz por harina de arroz, afrecho de yuca y harina de plátano disminuyó $(p<0,05)$ la postura y la conversión alimenticia por docena y $\mathrm{kg}$ de huevo producido.

Tabla 2. Parámetros productivos de codornices alimentadas con ingredientes alternativos.

\begin{tabular}{|c|c|c|c|c|c|}
\hline \multirow[b]{2}{*}{$\begin{array}{l}\text { Dietas experimentales } \\
\text { en substitución de } 50 \% \\
\text { del maíz }\end{array}$} & \multicolumn{5}{|c|}{ Parámetros productivos } \\
\hline & $\begin{array}{c}\text { Postura, } \\
\%\end{array}$ & $\begin{array}{l}\text { Peso del } \\
\text { huevo, g }\end{array}$ & $\begin{array}{l}\text { Consumo } \\
\text { de ración, } \\
\mathrm{g}\end{array}$ & $\begin{array}{l}\text { Kg de } \\
\text { ración/ } \\
\text { docena } \\
\text { huevos }\end{array}$ & $\begin{array}{c}\text { Kg de } \\
\text { ración / kg } \\
\text { de huevo }\end{array}$ \\
\hline Basal & $78,63 a$ & 10,23 & 24,39 & $0,376 \mathrm{c}$ & $3,057 \mathrm{c}$ \\
\hline Arroz partido & $66,26 a b$ & 10,31 & 24,11 & $0,440 \mathrm{bc}$ & $3,561 \mathrm{bc}$ \\
\hline Harina de arroz & $60,75 b$ & 10,20 & 24,54 & $0,491 a b$ & $4,004 a b$ \\
\hline Afrecho de yuca & $53,56 \mathrm{~b}$ & 10,01 & 24,01 & 0,545 a & 4,529 a \\
\hline Harina de plátano & $58,87 \mathrm{~b}$ & 10,23 & 24,80 & $0,514 a b$ & $4,180 a b$ \\
\hline$(P)$ & 0,02 & 0,38 & 0,06 & 0,01 & 0,02 \\
\hline $\mathrm{CV}^{1}, \%$ & 12,44 & 2,34 & 1,81 & 11,69 & 10,75 \\
\hline $\mathrm{SEM}^{2}$ & 0,05 & 0,15 & 0,28 & 0,04 & 0,26 \\
\hline
\end{tabular}

Fuente de consulta: autores. Letras distintas en la misma columna indican diferencia significativa por la Prueba de comparación de medias Scheffe $(p<0,05) \cdot{ }^{1}$ coeficiente de variación. ${ }^{2}$ desviación estándar de la media

La relativa baja producción de huevos de todos los tratamientos se debe al periodo experimental, dado que, al inicio del ciclo productivo, la postura es baja y luego se incrementa con la edad del ave (Zita et al. 2013) hasta alcanzar el pico máximo, que se presenta después del periodo en que se realizó este trabajo. Las dietas proporcionaban los requerimientos nutricionales, en las raciones conteniendo afrecho de yuca y harina de plátano puede haber influido en el aprovechamiento de los nutrientes la granulometría de estos ingredientes.

La producción de huevos obtenida en este trabajo fue inferior a la descrita por Pereira et al. (2016) y por Rosario y Nieves (2015) que verificaron máxima postura con 22 \% de proteína y consumo de ración de $29 \mathrm{~g}$, observando correlación positiva de la concentración de este nutriente en la ración y su consumo, con la tasa de postura. 
El mayor consumo diario de ración fue constatado con harina de plátano, seguido en orden descendente por harina de arroz, ración basal, arroz partido y afrecho de yuca. Ración basal presentó la mejor conversión alimenticia $(P<0,05)$. El consumo de ración es similar al obtenido por Filgueira et al. (2014) que constataron que el arroz partido no afecta el consumo.

Otra posible explicación a los resultados de consumo de ración en este trabajo comparado con los valores de Degollado (2018) y Silva et al. (2010) utilizaron diferentes niveles de energía y proteína, se debe a la influencia de la energía metabolizable sobre el consumo lo que explica que las aves ajustan la ingesta de las raciones para atender sus exigencias energéticas.

El peso medio del huevo fue superior al reportado por Degollado (2018) y Pino et al. (2018) utilizando harina de moringa y de pescado, respectivamente e inferior al obtenido por Barreto et al. (2010) incluyendo soya integral.

Los tratamientos con ración basal, con arroz partido, harina de arroz y harina de plátano contienen ligeramente mayores niveles de proteína bruta comparada con el afrecho de yuca, tratamiento que proporcionó menor producción y peso del huevo influenciando la conversión alimenticia por docena y por $\mathrm{kg}$ de huevo producido $(p<0,05)$, valores que difieren de Barreto et al. (2010); Pereira et al. (2016) y Degollado (2018), quienes verificaron mejores valores de conversión alimenticia en docenas de huevo y $\mathrm{kg}$ de masa de huevo por $\mathrm{kg}$ de ración, resultados que se pueden explicar por el elevado consumo de ración y menor peso y producción de huevos obtenidos en este experimento.

La menor conversión alimenticia $(p<0,05)$ de $\mathrm{kg}$ de ración/docenas de huevo y $\mathrm{kg}$ de ración/kg de huevo, se obtuvo con la dieta basal seguida de los tratamientos con substitución parcial de maíz por arroz partido, harina de arroz, harina de plátano y afrecho de yuca.

Los resultados de conversión alimenticia indican que los nutrientes son mejor aprovechados por las aves para producir un kg de masa de huevo y una docena de huevos por kg de ración. Silva et al. (2010) obtuvieron mejores resultados de conversión alimenticia con inclusión hasta del $12 \%$ de heno de la parte aérea de la yuca en la dieta para codornices de 60 a 144 días de edad.

\section{CONCLUSIONES}

El arroz partido puede sustituir en $50 \%$ al maíz, en la fabricación de raciones para codornices japonesas en fase inicial de postura sin afectar la producción de huevos.

El afrecho de yuca, las harinas de arroz y de plátano en sustitución al $50 \%$ del maíz, proporcionan bajos índices de desempeño productivo. 


\section{CONTRIBUCIÓN DE LA AUTORÍA}

Ferney Domingo Agudelo: administrador del proyecto, investigación, análisis de datos, edición.

Víctor Libardo Hurtado Nery: investigación, borrador original, revisión y edición.

Revisión, edición y publicación

Diana Milena Torres Novoa: revisión, edición, trámites de publicación.

\section{AGRADECIMIENTOS}

A la Universidad de los Llanos por la contribución financiera y logística.

\section{REFERENCIAS}

Barreto, S. L. T., Moura, W. C. O., Reis, R. S., Hosoda, L. R., Maia, G. V. C., Pena, G. M. (2010). Soja integral processada em dietas para codornas japonesas em postura. Revista Brasileira de Zootecnia, 39(9), 1978-1983. https://doi.org/10.1590/S1516$\underline{35982010000900016}$

Campos GCM, Vega JA, sustitutos de maíz utilizados en la alimentación animal en Costa Rica. Nutrición Animal Tropical 10(2): 91-113 DOI: http://dx.doi.org/10.15517/nat.v10i2.27327.

Degollado, A. K. M. (2018). Efecto de la inclusión de Moringa oleífera Lam. en dietas de codorniz, sobre postura, utilización de energia, proteína metabolizable y calidad de huevo (tesis de maestria). Universidad Autónoma de Nuevo León. Monterrey, Nuevo León, México.

Farias, N. N. P., Freitas, E. R., Xavier, R. P. S., Braz, N. M., Tavares, T. C. L., Figueiredo, C. W. S., Fernandes, D. R., Nascimento, G. A. J. (2014). Farelo integral de arroz parboilizado submetido a armazenamento prolongado para alimentação de codornas de corte. Pesquisa Agropecuaria Brasileira, 49(6), 407-415. https://doi.org/10.1590/S0100-204X2014000600001

Filgueira, T. M. B., Freitas, E. R., Quevedo Filho, B., Fernandes, D. R. I., Watanabe, H., Oliveira, A. N. (2014). Corn Replacement by Broken Rice in Meat-Type Quail Diets. Revista Brasileira de Ciência Avícola, 16(4), 345-350. https://doi.org/10.1590/1516$\underline{635 \times 1604345-350}$

Gopinger, E., Moraes, P. O., Catalan, A. A. S., Xavier, E. G., Castro, M. L., Schafhauser, Jr. J. (2014). Whole rice in japanese quails' diet. Acta Scientiarium Animal Sciences, 36(4), 363-367. https://doi.org/10.4025/actascianimsci.v36i4.24504 
Hrnčár, C., Hanusová, E., Hanus, A., Bujko, J. (2014). Effect of genotype on egg quality characteristics of japanese quail (coturnix japonica). Slovak Journal Animal Science, 47(1), 6-11.

Instituto Geográfico Agustín Codazzi, IGAC. (2021). Mapas de Colombia (mapa topográfico).

Narváez, S., Toro, J. P., Giraldo, C. E. (2012). Digestibilidad de matérias primas energéticas extrusadas em la alimentación de codornices (Coturnix coturnix japonicus). Biosalud, $11(2), 59-69$.

Pereira, A. A., Ferreira, D. A., Griep, J. D. N., Batista, L. C., De Moura, A. S., De Lima, J. D. M. (2016). Raspa da mandioca para codornas em postura. Acta Veterinaria Brasilica, 10(2), 123-129. https://doi.org/10.21708/avb.2016.10.2.5510

Pino, J. G., Pino, H. E., Villa, P. M., Ruiz, G. J. (2018). Efecto de diferentes nivels dietéticos de harina de pescado sobre la producción y calidad de huevos de codornices. Revista Cumbres, 4(2), 77-90.

Rahman, M. M., Suniza Anis, B. M. S., Jennlelyn, A. J. (2019). Growth performance and carcass characteristics of japanese quail fed rations with inclusion of diferente levels of broken rice. Malaysian Journal of Animal Science, 22(2), 27-33.

Rosario, J., Nieves, D. (2015). Producción y calidad de huevos de codornices alimentadas com dietas com harina de resíduos aserrados de carnicerias. Revista Científica, $\operatorname{XXV}(2), 139-144$.

Rostagno, H. S., Albino, L. F. T., Hannas, M. I., Donzele, J. L., Sakomura, N. K., Perazzo, F. $G$ et al. (2017). Tabelas brasileiras para aves e suínos, Composição de alimentos e exigências nutricionais. 4a Ed. Universidade Federal de Viçosa. p. 488.

Silva, J. A. O., Pereira, A. A., Lima, C. B., Ferreira, D. A., Santos, A. F., Barbosa, J. P. M., Ramos, D. A.V., Kitaoka, M. P. (2010) Inclusão do Feno da Parte Aérea da Mandioca em Rações para Codornas Japonesas em Fase de Postura sobre o Desempenho Zootécnico. Revista Cientifica de Produção Animal, 12(2), 177-179.

Zita, L., Ledvinka, Z., Klesalová, L. (2013). The effect of the age of Japanese quails on certain egg quality traits and their relationships. Veterinarski Arhiv, 83 (2), 223-232. 